The presence of EBV DNA in the plasma of persons who do not have nasopharyngeal carcinoma is probably due to transient viral replication, and $70 \%$ of persons who test positive would test negative 4 weeks later. ${ }^{1}$ Our observations are consistent with the findings of previous studies indicating that EBV replication is more active in older age groups ${ }^{2}$ and in cold temperatures, ${ }^{3}$ possibly because of impaired immune responses. In particular, exposure to the cold has been shown to impair the interferoninduced antiviral response. ${ }^{4}$ Cigarette-smoke extract has also been shown to directly promote EBV replication in in vitro experiments. ${ }^{5}$ In the context of screening for nasopharyngeal carcinoma, the presence of detectable EBV DNA in the plasma of persons who do not have the condition would represent false positive results that lead to further investigations, including endoscopic examination and magnetic resonance imaging. Therefore, screening may be more cost-effective if it is performed in the summer.
K.C. Allen Chan, F.R.C.P.A., Ph.D.

Sam W.I. Chu, B.Sc.

Y.M. Dennis Lo, F.R.S.

Chinese University of Hong Kong

Hong Kong, China

allen@cuhk.edu.hk

Supported by the Kadoorie Charitable Foundation and the Research Grants Council of Hong Kong.

Disclosure forms provided by the authors are available with the full text of this letter at NEJM.org.

1. Chan KCA, Woo JKS, King A, et al. Analysis of plasma EpsteinBarr virus DNA to screen for nasopharyngeal cancer. N Engl J Med 2017;377:513-22.

2. Thomasini RL, Pereira DS, Pereira FSM, et al. Aged-associated cytomegalovirus and Epstein-Barr virus reactivation and cytomegalovirus relationship with the frailty syndrome in older women. PLoS One 2017;12(7):e0180841.

3. Magrath IT, Pizzo PA, Novikovs L, Levine AS. Enhancement of Epstein-Barr virus replication in producer cell lines by a combination of low temperature and corticosteroids. Virology 1979;97:477-81. 4. Boonarkart C, Suptawiwat O, Sakorn K, Puthavathana P, Auewarakul P. Exposure to cold impairs interferon-induced antiviral defense. Arch Virol 2017;162:2231-7.

5. Xu FH, Xiong D, Xu YF, et al. An epidemiological and molecular study of the relationship between smoking, risk of nasopharyngeal carcinoma, and Epstein-Barr virus activation. J Natl Cancer Inst 2012;104:1396-410.

DOI: $10.1056 /$ NEJMc1800433

\title{
Letermovir Prophylaxis for Cytomegalovirus
}

TO THE EDITOR: Marty and colleagues (Dec. 21 issue $^{1}$ found that letermovir prophylaxis after hematopoietic-cell transplantation resulted in a lower risk of clinically significant cytomegalovirus (CMV) infection than placebo. This trial shows that a prophylactic agent can provide meaningful benefit after hematopoietic-cell transplantation, without considerable myelotoxic events.

Ganciclovir prophylaxis after hematopoieticcell transplantation reduces the risk of earlyonset CMV infection and disease. ${ }^{2}$ However, it delays the recovery of CMV-specific T-cell immunity, ${ }^{3}$ which increases the risk of delayed-onset CMV disease after the discontinuation of prophylaxis. ${ }^{4}$ Similarly, the reconstitution of CMVspecific immunity after hematopoietic-cell transplantation reduces the frequency of CMV reactivation and possibly disease. ${ }^{5}$ Therefore, monitoring of virus-specific immunity can help in tailoring the duration of prophylaxis.

Would the authors comment on whether, during the clinical implementation of letermovir prophylaxis, the duration of prophylaxis could be tailored on the basis of the development of CMV-specific T-cell-mediated immunity? Patients with delayed reconstitution of immunity could perhaps benefit from longer prophylaxis. In contrast, some patients might have their CMV-specific immunity reconstituted before day 100 after hematopoietic-cell transplantation, which may allow for the earlier cessation of prophylaxis, thereby avoiding cost and decreasing the likelihood of resistance. A similar strategy has been proposed in solid-organ transplantation.

Arnaud G. L'Huillier, M.D.

Atul Humar, M.D.

Deepali Kumar, M.D.

University Health Network

Toronto, ON, Canada

arnaud.Ihuillier@uhnresearch.ca

Dr. Humar reports receiving grant support from Qiagen, Astellas Pharma, Merck, and Roche; and Dr. Kumar, receiving grant support and consulting fees from Qiagen and Oxford Immunotec and grant support from Merck and Roche. No other potential conflict of interest relevant to this letter was reported.

1. Marty FM, Ljungman P, Chemaly RF, et al. Letermovir prophylaxis for cytomegalovirus in hematopoietic-cell transplantation. N Engl J Med 2017;377:2433-44.

2. Goodrich JM, Bowden RA, Fisher L, Keller C, Schoch G, Meyers JD. Ganciclovir prophylaxis to prevent cytomegalovirus disease after allogeneic marrow transplant. Ann Intern Med 1993;118:173-8. 
3. Li CR, Greenberg PD, Gilbert MJ, Goodrich JM, Riddell SR. Recovery of HLA-restricted cytomegalovirus (CMV)-specific T-cell responses after allogeneic bone marrow transplant: correlation with CMV disease and effect of ganciclovir prophylaxis. Blood 1994;83:1971-9.

4. Boeckh M, Leisenring W, Riddell SR, et al. Late cytomegalovirus disease and mortality in recipients of allogeneic hematopoietic stem cell transplants: importance of viral load and T-cell immunity. Blood 2003;101:407-14.

5. Tey SK, Kennedy GA, Cromer D, et al. Clinical assessment of anti-viral CD8+ T cell immune monitoring using QuantiFERONCMV assay to identify high risk allogeneic hematopoietic stem cell transplant patients with CMV infection complications. PLoS One 2013;8(10):e74744.

DOI: $10.1056 /$ NEJMc1800572

TO THE EDITOR: Marty et al. found that, among recipients of allogeneic hematopoietic-cell transplantation, letermovir prophylaxis resulted in a lower risk of clinically significant CMV infection than placebo. They also found that all-cause mortality was lower in the letermovir group than in the placebo group through week 24 (10.2\% vs. $15.9 \%, \mathrm{P}=0.03)$ but not through week 48 (20.9\% and $25.5 \%$, respectively; $\mathrm{P}=0.12$ ).

In previous work, we and colleagues found that CMV reactivation after allogeneic hematopoietic-cell transplantation was associated with a reduced risk of relapse among patients with acute myeloid leukemia (AML). ${ }^{1}$ Although the mechanism is not fully elucidated, other reports have also shown that immunologic activation against CMV reactivation may play a role in reducing the risk of early relapse, with or without a beneficial effect on overall survival. ${ }^{2-4}$ If letermovir prophylaxis, administered as planned in the protocol, was associated with an increased risk of relapse by suppressing CMV reactivation, alternative strategies, such as preemptive therapy or shorter prophylaxis, might result in a better outcome, depending on the specific disease or disease status. We therefore ask that the authors provide data about the association between disease risk and the incidence of relapse.

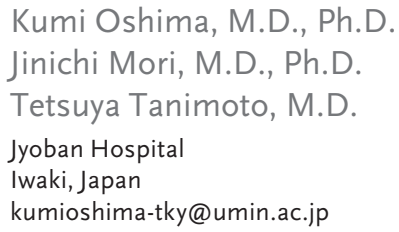

No potential conflict of interest relevant to this letter was reported.

1. Takenaka K, Nishida T, Asano-Mori Y, et al. Cytomegalovirus reactivation after allogeneic hematopoietic stem cell transplantation is associated with a reduced risk of relapse in patients with acute myeloid leukemia who survived to day 100 after transplantation: the Japan Society for Hematopoietic Cell Transplantation Transplantation-related Complication Working Group. Biol Blood Marrow Transplant 2015;21:2008-16.

2. Elmaagacli AH, Steckel NK, Koldehoff M, et al. Early human cytomegalovirus replication after transplantation is associated with a decreased relapse risk: evidence for a putative virus-versusleukemia effect in acute myeloid leukemia patients. Blood 2011; 118:1402-12.

3. Teira P, Battiwalla M, Ramanathan M, et al. Early cytomegalovirus reactivation remains associated with increased transplantrelated mortality in the current era: a CIBMTR analysis. Blood 2016;127:2427-38.

4. Green ML, Leisenring WM, Xie H, et al. CMV reactivation after allogeneic HCT and relapse risk: evidence for early protection in acute myeloid leukemia. Blood 2013;122:1316-24.

DOI: $10.1056 /$ NEJMc1800572

TO THE EDITOR: Marty et al. report the results of a randomized, double-blind, placebo-controlled, superiority trial of letermovir prophylaxis to prevent $\mathrm{CMV}$ reactivation in patients who had undergone hematopoietic-cell transplantation. The primary end point was the proportion of patients who had clinically significant CMV infection through week 24 after transplantation. Letermovir prophylaxis resulted in a lower risk of clinically significant CMV infection than placebo. The authors report data on CMV DNA levels, antiviral treatment, and CMV DNA sequence analysis in the patients at week 14 after transplantation (Table 2 of the article; and Table S7 in the Supplementary Appendix, both available at NEJM.org). However, virologic details and data about CMV DNA sequence analysis in the 52 patients who had received letermovir prophylaxis and who had a clinically significant CMV infection at week 24 that was treated with preemptive therapy are not shown. In vitro studies suggest that letermovir has a low genetic barrier to the development of letermovir resistance and that it is important to monitor viral isolates obtained from patients treated with letermovir. ${ }^{1,2}$ Because limited information is currently available from clinical studies, we think that these aspects should be clarified.

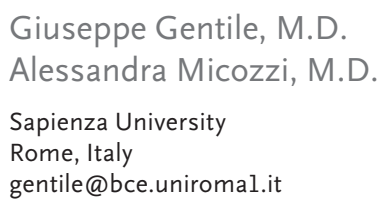

No potential conflict of interest relevant to this letter was reported. 
1. Chou S. Rapid in vitro evolution of human cytomegalovirus UL56 mutations that confer letermovir resistance. Antimicrob Agents Chemother 2015;59:6588-93.

2. Piret J, Goyette N, Boivin G. Drug susceptibility and replicative capacity of multidrug-resistant recombinant human cytomegalovirus harboring mutations in UL56 and UL54 genes. Antimicrob Agents Chemother 2017;61(11):e01044-e17.

DOI: $10.1056 /$ NEJMc1800572

THE AUTHORS REPLY: We agree with L'Huillier and colleagues that tailoring prophylaxis to the dynamic risk of CMV reactivation and disease in a diverse population of patients who have undergone allogeneic hematopoietic-cell transplantation is ideal. The trial targeted a well-known period of CMV risk through day 100 (week 14) after transplantation, following a clinical trial design similar to that used in kidney transplantation. ${ }^{1}$ There was a higher number of CMV events after the prophylaxis period among the patients who were at higher risk for CMV infection than among those at lower risk (Fig. 2B and 2C of our article), which indeed suggests that a longer period of letermovir prophylaxis, or the reinstitution of prophylaxis during new risk periods (e.g., graftversus-host disease), would be clinically meaningful in certain patients. Although some assays are available to measure certain aspects of CMVspecific T-cell-mediated immunity, their specificity and clinical usefulness in determining when to stop antiviral prophylaxis or stop CMV surveillance for preemptive treatment purposes have yet to be determined in patients who have undergone hematopoietic-cell transplantation.

Oshima and colleagues inquire about $\mathrm{AML}$ relapse events and the effect of preventing CMV reactivation with letermovir on these events. Among patients who underwent hematopoieticcell transplantation for AML in our trial, 11 of 142 patients (7.7\%) who received letermovir had AML relapse by week 14 , as compared with 8 of 72 (11.1\%) who received placebo $(\mathrm{P}=0.45)$ (Table 1 of our article, and Table S11 in the Supplementary Appendix of our article). The incidence of AML relapse through week 24 after transplantation was $14.1 \%$ (20 of 142 patients) in the letermovir group and 19.4\% (14 of 72) in the placebo group $(\mathrm{P}=0.33)$ (Table $\mathrm{S} 12$ in the Supplementary Appendix of our article). These results do not support a higher risk of AML relapse among patients who received letermovir prophylaxis to prevent $\mathrm{CMV}$ reactivation than among those who received placebo. These results align with the findings of Teira and colleagues, who did not find an association between CMV reactivation and AML relapse and who also noted that all-cause mortality before day 100 after hematopoieticcell transplantation was higher among patients with CMV reactivation than among those without CMV reactivation. ${ }^{2}$

Gentile and Micozzi inquire about sequencing details in patients who had clinically significant CMV infection (leading to preemptive treatment or in whom CMV disease developed) between the end of the letermovir prophylaxis period and week 24 after transplantation (32 patients) (Table 2 of our article). Letermovir genotypic resistance analysis was performed by polymerasechain-reaction amplification and sequencing of the coding regions of the CMV genes UL56 and UL89. DNA that included these CMV genes was isolated from plasma that was obtained at the time of the events. After the alignment of the deduced UL56-UL89 protein sequences to references from a letermovir-susceptible CMV strain, genotypic variants that were detected at a frequency of at least $5 \%$ of the sample sequence data at a given position were identified. No known letermovir-resistance mutations were found in 16 of these 32 patients who had genotyping results that could be evaluated.

Francisco M. Marty, M.D.

Dana-Farber Cancer Institute

Boston, MA

fmarty@bwh.harvard.edu

Johan Maertens, M.D., Ph.D.

Universitaire Ziekenhuizen Leuven

Leuven, Belgium

Cyrus Badshah, M.D., Ph.D.

Merck

Kenilworth, NJ

Since publication of their article, Dr. Marty reports receiving consulting fees from GlaxoSmithKline; and Dr. Badshah, holding stock in Merck. No further potential conflict of interest relevant to this letter was reported.

1. Humar A, Lebranchu Y, Vincenti F, et al. The efficacy and safety of 200 days valganciclovir cytomegalovirus prophylaxis in high-risk kidney transplant recipients. Am J Transplant 2010;10: 1228-37.

2. Teira P, Battiwalla M, Ramanathan M, et al. Early cytomegalovirus reactivation remains associated with increased transplant-related mortality in the current era: a CIBMTR analysis. Blood 2016;127:2427-38.

DOI: $10.1056 / N E J M c 1800572$ 